\title{
Balancing Quality, Cost, and Access During Delivery of Newer Cellular and Immunotherapy Treatments
}

\author{
Praveen Ramakrishnan Geethakumari ${ }^{1}$. Dheepthi Perumal Ramasamy ${ }^{1} \cdot$ Bhagirathbhai Dholaria $^{2} \cdot$ Jesús Berdeja $^{3}$. \\ Ankit Kansagra ${ }^{1}$ (B)
}

Accepted: 27 April 2021 / Published online: 5 June 2021

(C) The Author(s), under exclusive licence to Springer Science+Business Media, LLC, part of Springer Nature 2021

\begin{abstract}
Purpose of Review The chimeric antigen receptor (CAR) T-cell therapy is currently changing the landscape of hematologic malignancies with multiple FDA-approved cell therapy products in the USA. The current administration process of the CAR Tcell therapy is complicated, labor-intensive, and expensive.

Recent Findings The chimeric antigen receptor (CAR) T-cell therapy is currently changing the landscape of hematologic malignancies with multiple FDA-approved cell therapy products in the USA. The current administration process of the CAR T-cell therapy is complicated, labor-intensive, and expensive.

Summary This review article addresses the present-day challenges and discusses opportunities to optimize the access and affordability of the CAR T-cell therapy. The field of cellular immunotherapy is going to change the future of solid tumors and non-oncological diseases. However, this promising therapy poses challenges in the administration and management of quality in the current field of healthcare. We describe various novel approaches to manage challenges in improving access and improving widescale implementation of cellular therapies.
\end{abstract}

Keywords Immunotherapy $\cdot$ CAR T cell therapy $\cdot$ Immune-effector cell therapy $\cdot$ Lymphoma $\cdot$ Multiple myeloma $\cdot$ Acute lymphoblastic leukemia $\cdot$ Quality $\cdot$ Barriers $\cdot$ Access $\cdot$ Cost-effectiveness

\section{Introduction}

\section{The Current Landscape of Cellular and Immunotherapy}

Advances in immunotherapy have transformed the landscape and cemented its role as the fourth pillar in oncologic therapeutics besides surgery, cytotoxic chemo-, and radiation therapy

Praveen Ramakrishnan Geethakumari and Dheepthi Perumal Ramasamy contributed equally to this work.

This article is part of the Topical Collection on Health Economics

Ankit Kansagra

ankit.kansagra@utsouthwestern.edu

1 Simmons Comprehensive Cancer Center, University of Texas Southwestern Medical Center, 2201 Inwood Road, Dallas, TX 76034, USA

2 Vanderbilt University Medical Center, Nashville, TN, USA

3 Sarah Cannon Research Institute and Tennessee Oncology, Nashville, TN, USA
[1]. Engineered cellular therapies, including chimeric antigen receptor $\mathrm{T}$ cell (CAR $\mathrm{T}$ cell) therapy, have been a gamechanger in hematologic malignancies [2]. There are currently four CD19-directed autologous second-generation CAR T cell products approved by the US Food and Drug Administration (FDA) for the treatment of relapsed or refractory (R/R) lymphoid malignancies. Tisagenlecleucel (Tisa-cel, KYMRIAH®, Novartis Pharmaceuticals Corp.) was first approved in 2017 for treating R/R acute lymphoblastic leukemia (ALL) [3••]. This was followed by axicabtagene ciloleucel (Axi-cel, YESCARTA®, Kite Pharma, a Gilead Company) (2017) [4•], tisa-cel (2018) [5], and liso-cel (Liso-cel, BREYANZI ${ }^{\circledR}$, Bristol Myers Squibb Corp.) (2021) [6] being approved for treating $\mathrm{R} / \mathrm{R}$ aggressive large $\mathrm{B}$ cell lymphomas. Brexu-cel (Brexu-cel, TECARTUS $®$, Kite Pharma, a Gilead Company) was approved (2020) for the treatment of R/R mantle cell lymphoma [7]. Table 1 describes all the FDA-approved CAR T cell therapies, their indications and wholesale acquisition cost.

CAR $T$ cell therapies targeting CD19 have demonstrated efficacy across the spectrum of indolent B cell lymphomas, including chronic lymphocytic leukemia (CLL) [8], follicular, 
Table 1 FDA-approved CAR T cell therapies

\begin{tabular}{|c|c|c|c|}
\hline Name & Indication & Administration and dosing & Cost (US dollar) \\
\hline Kymriah (Tisagenlecleucel) & $\begin{array}{l}\text { - Patients up to } 25 \text { years of age with } \\
\text { B cell precursor ALL that is } \\
\text { refractory or in 2nd or later relapse } \\
\text {-Adults with relapsed/refractory large } \\
\text { B cell lymphoma (including DLBCL, } \\
\text { high grade B cell lymphoma, and } \\
\text { DLBCL arising from follicular } \\
\text { lymphoma) after two or more lines } \\
\text { of prior therapy }\end{array}$ & $\begin{array}{r}- \text { Single dose of } 0.2-5.0 \times 10^{6} \\
\text { CAR T cells } / \mathrm{kg}(\leq 50 \mathrm{~kg}) \\
\text { - Single dose of } 0.1-2.5 \times 10^{6} \\
\text { CAR T cells } / \mathrm{kg}(>50 \mathrm{~kg})\end{array}$ & $\begin{array}{l}\$ 475,000 \text { (pediatric and } \\
\text { young adult ALL) } \\
\$ 373,000 \text { (large cell } \\
\text { lymphoma) }\end{array}$ \\
\hline Yescarta (Axicabtageneciloleucel) & $\begin{array}{l}\text { - Adult patients with relapsed/refractory } \\
\text { large B cell lymphoma after two } \\
\text { or more lines of prior therapy }\end{array}$ & $\begin{array}{l}\text { - Single dose of } 2 \times 10^{6} \text { CAR T } \\
\text { cells } / \mathrm{Kg} \text { (maximum of } 2 \times 10^{8} \\
\text { CAR T cells) }\end{array}$ & $\$ 373,000$ \\
\hline Breyanzi (Lisocabtagenemaraleucel) & $\begin{array}{l}\text { - Adult patients with relapsed/refractory } \\
\text { large B cell lymphoma (including } \\
\text { DLBCL, high grade B cell lymphoma, } \\
\text { primary mediastinal large B cell } \\
\text { lymphoma, and follicular lymphoma } \\
\text { grade 3B) after } 2 \text { or more lines of } \\
\text { prior therapy }\end{array}$ & $\begin{array}{l}- \text { Single dose of } 50-110 \times 10^{6} \\
\text { CAR T cells (consisting of } \mathrm{CD}^{8} \\
\text { and CD4 component) }\end{array}$ & $\$ 410,300$ \\
\hline Tescartus (Brexucabtageneautoleucel) & $\begin{array}{l}\text { - Adult patients with relapsed/refractory } \\
\text { mantle cell lymphoma }\end{array}$ & $\begin{array}{l}\text { - Single dose of } 2 \times 10^{6} \mathrm{CAR} \\
\mathrm{T} \text { cells } / \mathrm{kg} \text { (maximum of } \\
2 \times 10^{8} \mathrm{CAR} \mathrm{T} \text { cells) }\end{array}$ & $\$ 373,000$ \\
\hline ABECMA (Idecabtagenevicleucel) & $\begin{array}{l}\text { - Adult patients with relapsed/refractory } \\
\text { multiple myeloma after four or more } \\
\text { lines of therapy (including an } \\
\text { immunomodulatory agent, a proteasome } \\
\text { inhibitor, and an antiCD38 } \\
\text { monoclonal antibody) }\end{array}$ & $\begin{array}{l}\text { - Single dose of } 300-460 \times \\
10^{6} \text { CAR T cells }\end{array}$ & $\$ 419,500$ \\
\hline
\end{tabular}

and marginal zone lymphomas [9]. Cellular therapies targeting B cell maturation antigen (BCMA) have shown impressive and durable responses in treating $\mathrm{R} / \mathrm{R}$ multiple myeloma with the most recent FDA approval of ide-cel (Ide-cel, ABECMA®, Bristol Myers Squibb Corp) $[10,11]$. The field of cellular therapy is witnessing an explosion of novel and promising therapies, to the extent that the FDA predicts the development of $>200$ investigational new drugs per year and expects approval for 10-20 cellular and gene therapy products by 2025 [12].

Emerging long-term follow-up data shows the transformative potential of cellular therapies' as a curative option for patients with $\mathrm{R} / \mathrm{R} \mathrm{B}$ cell malignancies [13-17]. Despite this data, only a small proportion of eligible patients are currently able to successfully receive CAR T cell therapy. Thus, the field poses several challenges: balancing quality and efficiency, cost, access, and expertise needed to manage unique immune effector cell-related toxicities. Currently, cellular therapies are being delivered to patients mostly by experienced teams at designated centers of excellence (COE) who routinely perform stem cell transplantation. CAR T cell therapies have a high price tag at $\$ 373,000$ to $\$ 475,000 /$ dose, leading to restrictions in insurance coverage and reimbursement. This poses challenges in improving affordability and access to these cellular therapies for all eligible patients. Analyzing these challenges and developing practical solutions is imperative as the field continues to grow with safer and more effective treatment options that ultimately could be delivered across a broader spectrum of practice locations [18-20, 21••, 22, 23]. This review describes the currently approved CAR $\mathrm{T}$ cell therapies for hematologic malignancies and the challenges and opportunities in balancing quality, cost, and access in the effective delivery of current and future cellular therapies.

\section{Practical Aspects in Clinical Administration and Challenges of Current Autologous CAR T Cell Products}

The current manufacturing and delivery of commercial autologous CAR T cell products are resource, and labor-intensive. The process and quality measures were initially designed based on algorithms of stem cell transplant programs [24••]. Engineered cellular therapies are associated with unique acute (30 days) and late phase (beyond 30 days) toxicities. Toxicities in the acute phase include cytokine release syndrome (CRS), immune effector cell-associated neurotoxicity syndrome (ICANS), infections, and cytopenias. These are potentially life-threatening and require close monitoring and 
multidisciplinary management. Most acute phase management is currently done as an inpatient but with safer products, more outpatient management is likely in the future. Late phase toxicities include persistent or recurrent cytopenias which necessitates growth factor and/or transfusion support, and prolonged immune suppression leading to recurrent infections. Thus, infection prophylaxis, immunoglobulin replacement, and revaccination are the issues in this phase [25]. Due to the logistical differences compared to the standard autologous stem cell transplantation process, the processes needed modification, and therefore many centers created immune effector cell (IEC) paradigms.

\section{Steps Involved in the Design of an IEC Program}

A patient's journey through an IEC program and CAR T cell therapy can be summarized in eight steps as described in Fig. 1 (adapted from Berdeja et al. [26]): Fig. 1 describes the patients CAR T cell therapy journey and associated barriers.

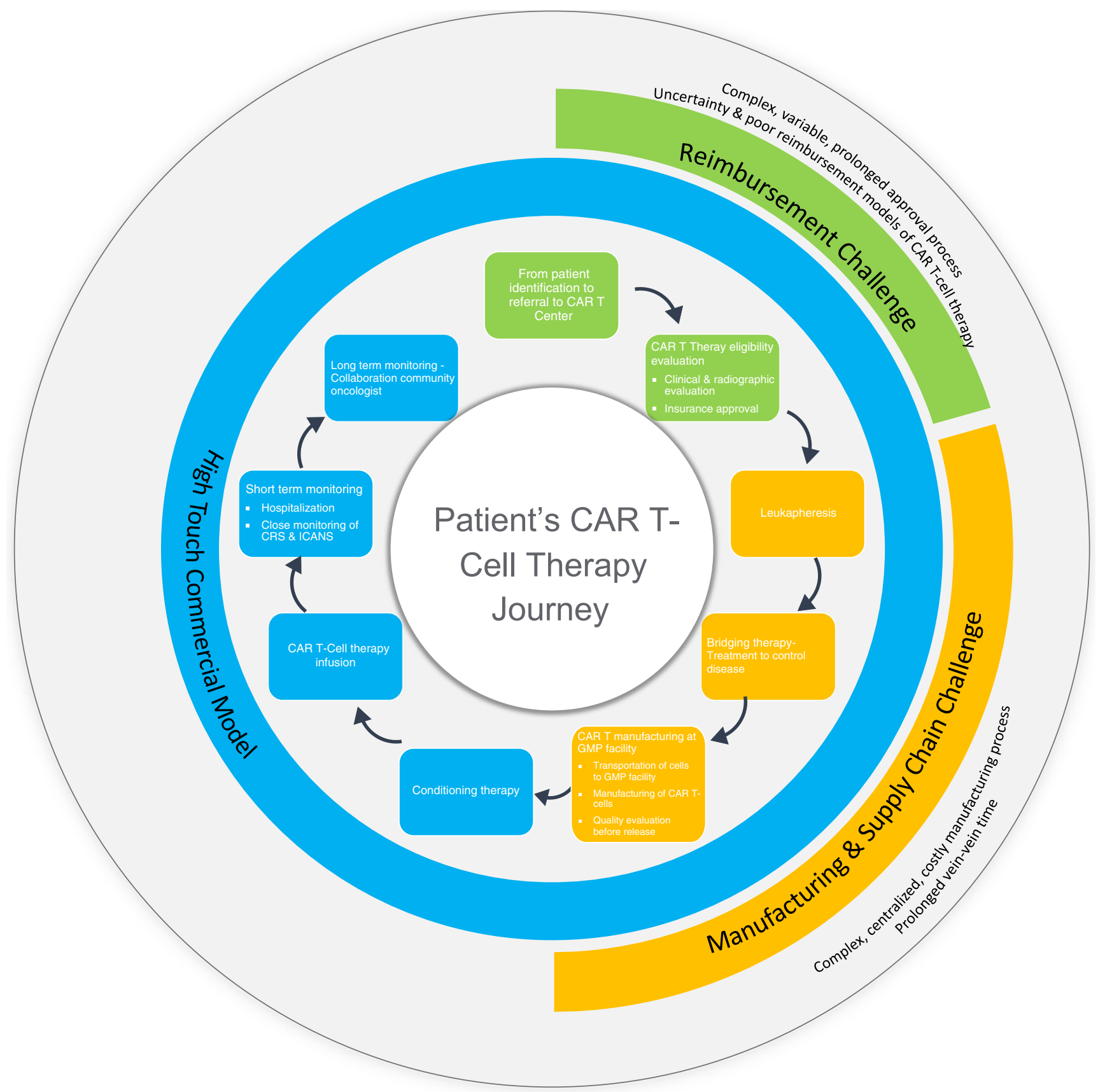

Fig. 1 Patients CAR T cell therapy journey and associated barriers 
1. Intake and triage. The process is initiated by either the patient or a referring provider. Ideally, this should involve a dedicated triage navigator or nurse, CAR T coordinator, and financial coordinator to assess insurance coverage.

2. Consultation with an IEC provider. This is to assess eligibility for a CAR T cell product. If found eligible, the CAR T coordinator sets in quick motion all necessary workup and communication with other parties, including apheresis staff (to determine the type of apheresis access needed and timing), social work evaluation for compliance, caregiver support, financial resource, and local housing requirements if required.

3. CAR T cell manufacture. This step involves the leukocyte apheresis, processing, shipping, and manufacturing of cells. The "vein-to-vein" time varies but, on average, takes from 3 to 6 weeks. Once the CAR T product is manufactured, it is shipped and received by the cell processing laboratory in preparation for infusion.

4. Bridging therapy. While awaiting the manufacture of CAR T cells, many patients with aggressive malignancies will need bridging therapy to control the disease. This can be delivered by the IEC provider or referring oncologist.

5. Re-evaluation before lymphodepletion and CAR T cell therapy. Once the product is ready for infusion, the patient is re-evaluated to ensure eligibility and no issues have arisen in the interim, including deterioration in performance status, organ function, or infections. If eligible, the IEC team proceeds with lymphodepleting chemotherapy, usually delivered over 3 days and done on an outpatient basis. The patient then undergoes infusion of the CAR T cell product, usually 2-7 days after lymphodepletion.

6. Early monitoring phase after CAR T cells (first 30 days). The patient is at the highest risk for developing CRS, ICANS, and pancytopenia during the first 2 to 3 weeks after CAR $\mathrm{T}$ cell infusion with currently available CAR products. Multidisciplinary coordination is organized the IEC physician, with the intensive care team, neurology, emergency physicians, and other consultants as needed. An experienced nursing and pharmacy team is paramount through this process. For CRS management, emergency availability of anti-cytokine agents, like tocilizumab and anakinra, should be ensured. Generally during this phase, the patient and caregiver stay within a short driving distance from the IEC center.

7. Long-term surveillance. At day +30 , after initial imaging and disease response assessment, the patient could be transitioned back to the referring oncologist for ongoing follow-up. The patients in the late phase may need ongoing monitoring for disease relapse, infection prophylaxis, transfusion and/or growth factor support, immunizations, and intravenous immunoglobulin infusions. Continued communication between the primary oncologist and IEC provider is important.

8. Data management, regulatory issues, and outcome reporting. Continuous outcome reporting is mandated by the Centers for Medicare and Medicaid (CMS). Most centers in the USA are now reporting IEC data to the Center for International Blood and Marrow Transplant Research (CIBMTR).

\section{Quality and Efficiency of IEC Therapies: Stakeholders and Outcomes}

Several stakeholders are at play in ensuring patients get the highest quality care with the best outcomes from engineered cellular therapies [27].

1. Patients. They are the prime focus for all quality and efficiency measures during the cellular therapy process. The best possible outcomes expected by undergoing this process include survival, disease control, low rate of complications, improved quality of life, and a high degree of satisfaction with the care received. They also aim for affordable care without undue financial toxicity. Socio-demographic and disease-related factors can also impact the quality and efficiency of care.

2. Referring physicians. The hematologist-oncologists who refer their patients to an IEC center desire for the described positive outcomes for their patients. They also value effective communication, ease of access to the IEC center, and center reputation.

3. IEC center/physician. The treating IEC center and physician desire to improve their outcomes, foster quality research and be financially viable.

4. Payers. Private and government payers have similar interests, and they are interested in high-value affordable care. They look for objective metrics of quality and efficiency of care delivery, such as accreditation by FACT (Foundation for Accreditation for Cellular Therapy), center volume, survival rates, resources available to the center, and ability to deliver care for complex patients.

5. Professional and patient organizations. Professional societies like the American Society for Transplantation and Cellular Therapy (ASTCT) can be considered a stakeholder in ensuring high-value patient care through their initiatives focusing on improving quality and evidence-based guidelines providers for optimal care of patients receiving IEC therapy. Patient advocacy organizations can also be included as they are involved in providing resources, support, and education to the patients. 


\section{Existing Mechanisms for Optimizing the Quality of Patient Care During IEC Therapy: Lessons from Stem Cell Transplantation}

The following are the specific established quality standards and measures that can be adapted from hematopoietic stem cell transplantation (HSCT) [26-28]:

1. Structural indicators. Centers providing cellular therapy need a robust clinical infrastructure to handle the complex scheduling logistics, maintain the "chain-of-custody" and "chain-of-identity" of the cellular product, and facilitate communication to manage potentially severe toxicities. Cellular therapies are also different from other oncology drugs, as they are "living drugs" and must be temperature controlled at all times and can be manipulated only under aseptic conditions. Structural indicators also focus on personnel and the need to have experienced clinical and nonclinical staff at the center. CMS has established a risk evaluation and mitigation strategy (REMS) program to define a center's criteria to qualify as an FDA-approved IEC center. Each center must establish standard-operating procedures that meet all REMS requirements, and all staff members involved in patient care during IEC therapy need to be trained and certified on a regular basis.

2. Process indicators. Measures in these categories capture variables related to the actual delivery of cellular therapy.

3. Outcome indicators. The most accepted metric is overall survival. Other measures include disease control, complication rates, and quality of life.

Emphasis on quality measures has been shown to improve allogenic HSCT outcomes. A 3-tiered hierarchical model proposed by Porter et al. can focus on outcomes based on quality metrics, with the top tier focusing on overall survival, the second tier on the process and time to recovery, and the third tier on the sustainability and long-term consequences of therapy [29]. FACT standards for HSCT cover three areas: (1) clinical programs, (2) collection of cellular products, and (3) cellular processing. HSCT standards are updated and jointly published with the Joint Accreditation Committee ISCTEurope and EBMT (JACIE). Achieving FACT accreditation requires a comprehensive inspection and maintenance every 3 years, which demonstrates that a program is committed to maintaining quality measures in cell therapy practices and high-value patient care.

In 2017, FACT formulated the Standards and Accreditation Program for IEC therapy with a task force representing FACT, ISCT, American Society of Gene and Cellular Therapy (ASGCT), Society for Immunotherapy of Cancer (SITC), and cellular therapists from 10 academic centers. IEC was defined as "cells used to modulate, elicit, or mitigate an immune response for therapeutic intent," including the dendritic, natural killer, T, or B cells. The published FACT IEC standards guide in 4 areas in addition to standard transplant guidelines [28]:

(a) Location of cell manufacturing. The IEC program under FACT is only responsible for the steps that they are directly involved in. Manufacturing of the product may happen at a third-party site and agreement on chain-ofcustody through multiple handoffs until infusion needs to be reached.

(b) Identification and management of CRS. This pertains to REMS training of all providers involved in care of patients receiving IEC therapy. The training involves detection of complications and demonstrating competency in management. Pharmacy formularies need to be adapted for using required supportive medications for CRS management.

(c) Coordination and education. The institution should demonstrate appropriate communication pathways between the many providers involved and rapid escalation of care when needed.

(d) Data management and oversight. Designated staff should collect data on product safety, efficacy, and outcomes and be reviewed by the IEC program director yearly. The CIBMTR cellular therapy forms allow the collection of data for the whole field.

As many clinical site's teams overseeing IECs versus HSCT may be different. Hence, FACT accreditation for IECs is separate from that of HSCT, although there is significant overlap in standards for guidance and infrastructure. Outside the USA, additional regulatory oversight is required where these products fall into the ATMP (Advanced Therapy Medicinal Product) and must be handled according to the European Medicines Agency (EMA).

\section{Centers of Excellence (COE) and Their Role in Program Regulation and Quality Improvement [30]}

\section{Payer-Based COE Networks}

Many large payers have developed a panel of COE from HSCT data based on center volume as a mark of clinical experience, a requirement for society accreditation, and quality standards like risk-adjusted survival rates. Additional criteria include geographic location to increase patient access and limit travel. There is also a bonus for contracted care that promotes value-based care.

\section{Manufacturer Based "COE-Like" Cellular Therapy Networks}

The registrational trials of CAR T products were performed in less than 30 clinical centers, selected based on clinical 
research expertise for ALL and non-Hodgkin lymphoma (NHL), and most were from traditional allogeneic HSCT COE networks. With FDA approval of multiple products, manufacturers would want to increase the number of available sites while ensuring complication rates are low as outcomes data are being compared across sites and products.

\section{Quality and Efficiency in IEC Therapies: Challenges and Opportunities}

Despite existing mechanisms to ensure high-value and timely patient care, several challenges exist hindering the true potential and expansion of IEC therapies. From the knowledge gained from clinical HSCT practice [27, 30], there are potential opportunities as outlined in the following to address these challenges to optimize quality and efficiency as discussed as follows:

1. Timely referral to IEC center and education to mitigate knowledge gaps. There is significant under-referral of eligible patients from practicing oncologists to IEC centers (discussed in next section), primarily due to lack of knowledge of this relatively new therapy, its potential, and eligibility, especially in older or frail patients. In the transplant realm, $<30 \%$ of eligible myeloma patients are referred for autologous HSCT, and $<20 \%$ of acute myeloid leukemia patients are referred for allogeneic HSCT. Thus, professional societies and academic centers need to be actively involved in educational activities to dispel gaps in knowledge. Early referrals in relapsing disease states for IEC therapy evaluation will expand the eligible patient pool and outcomes.

2. Health disparities. Disparities in access and outcomes after IEC therapies are becoming apparent as more patients are being assessed and treated. Centers need to be equipped to engage internal and external resources in tackling issues like expanding insurance coverage, transportation, lodging, and ensuring culturally appropriate care [31].

3. Variation in resources and personnel. Currently, centers vary in infrastructure, capacity, and personnel for performing IEC therapies. Centers will have to continually innovate as the field evolves and adopt best practices from their peers.

4. Care coordination and transitions of care. Effective communication with referring providers throughout IEC therapy is essential for ensuring high-quality care. Technological advances, including improved electronic health records, should help smooth care transitions after the acute treatment phase is over.

5. Measurement of quality and efficiency. Novel data collection methods should focus not only on overall survival disease-free survival, IEC-related toxicities, but also on metrics like patient-reported outcomes [32] and quality of life. Long-term outcomes and delayed side effects post cellular therapy are being discerned and are areas of active research [33, 34].

6. Harmonization of quality measurement and reporting. Standardizing and simplifying reporting across IEC centers could avoid duplication and redundancy across multiple platforms.

\section{Optimizing Access and Affordability of CAR T Cell Therapy}

Despite very robust data in clinical trials, challenges affect commercial CAR T cell therapy's widespread implementation [35]. Cellular Immunotherapy Data Resource (CIDR), a national initiative from CIBMTR, collects data about cellular therapies' long-term safety and efficacy. Using SEERMedicare Database, it is anticipated that $\sim 3000$ patients/year with diffuse large cell lymphoma are potentially eligible for CAR T cell therapy (with currently approved indication). CIDR reported only 2581 patients who received CAR T Cell therapy for diffuse large $\mathrm{b}$ cell lymphoma (DLBCL) between 2016 and 2020 as reported by 148 centers in north America. This represents a considerable disparity in access to CAR T cell therapy. In general, the issues can be divided into two major categories - patient-related factors or product-related factors. Figure 2 describes approaches to overcome hurdles in improving access to CAR T cell therapy.

\section{Product-Related Factors}

\section{Reducing "Brain to Vein" Time}

Two significant hurdles exist in getting the patients to receive CAR T cell therapy. First is insurance hurdles to get CAR T cell therapy approved, and second is manufacturing it quickly to avoid delay in getting CAR T cell therapy to the patient.

Brain to vein is a term mentioned by Elnair and colleagues describing the time it takes for a physician plans to treat a patient with CAR $\mathrm{T}$ cell therapy to patient receiving CAR T cell infusion. Long brain to vein time is an extremely critical issue as CAR T centers have to go through a tedious insurance approval before initiation of apheresis leading to significant delays. Elnair et al., in a small retrospective institutional study, demonstrated that public insurance groups had a significantly shorter time of approval than private insurance ( $17 \mathrm{vs} .30$ days) [33]. Reducing the time it takes for insurance approval is an easy, modifiable factor with interventions like standardization of approval process to impact patient outcomes. Similarly institutions have an approval process where all pre-CAR $\mathrm{T}$ 


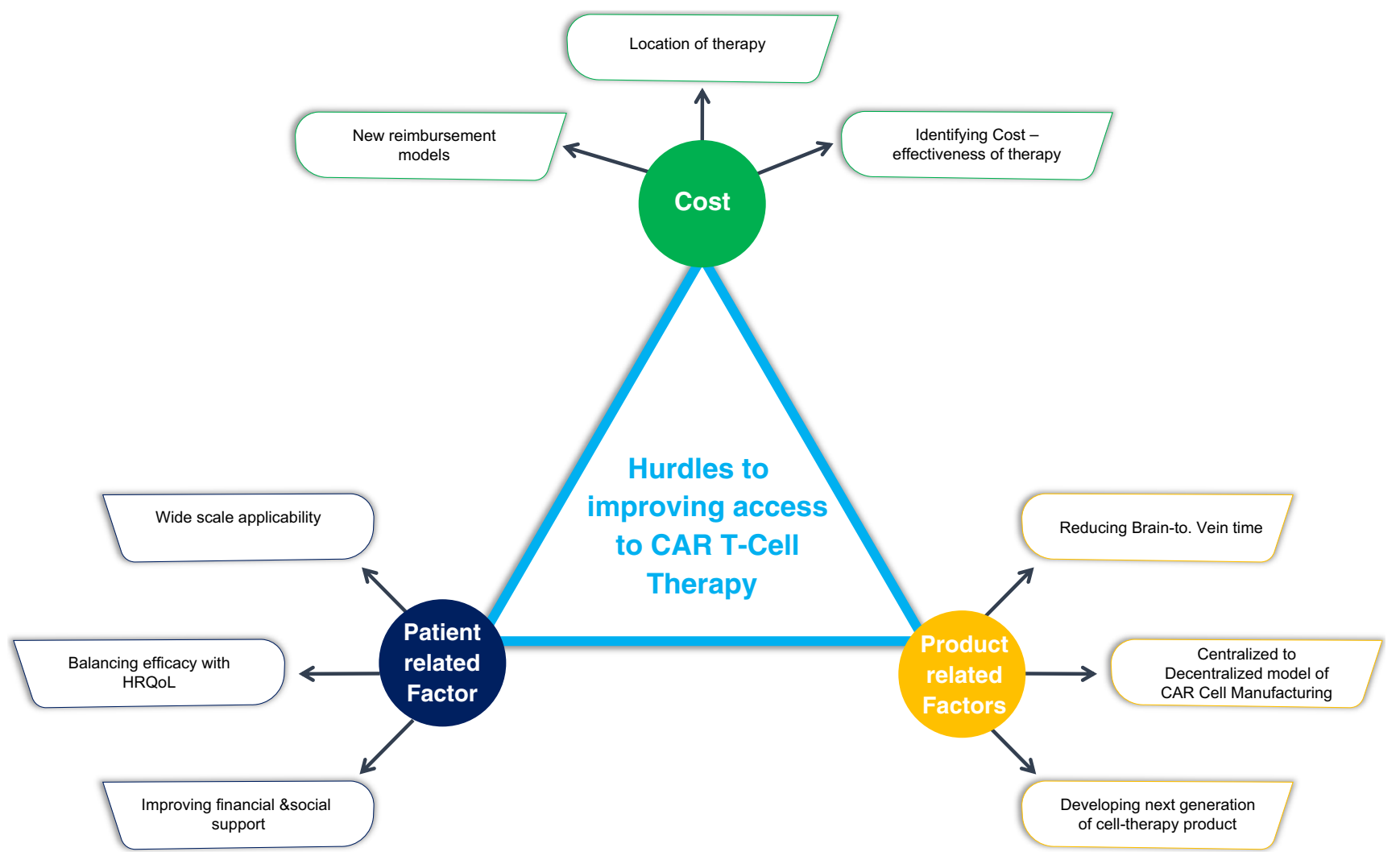

Fig. 2 Toolkit to overcomes hurdles in improving access to CAR T cell therapy

workup is performed and presented to an internal committee that can delay patients receiving CAR T cell therapy.

As described in Fig. 1, the most critical step in patient outcomes is "vein-to-vein" time which is defined as the time between apheresis, i.e., collecting $\mathrm{T}$ cells to infusion engineered product back to the patient. All FDA-approved products require $3-5$ weeks of manufacturing and quality assessment before the product is available for the patient. This long wait time commonly requires patients to have bridging therapy, which can lead to increased toxicity, and even post CAR T cell therapy complications [36]. Manufacturing companies are already exploring opportunities to overcome this challenge by optimizing their quality control process or developing novel T cell engineering methodologies [37].

\section{Decentralization of CAR T Cell Production}

Decentralizing CAR T cell production can provide a significant breakthrough in access to cellular therapies. Decentralizing can reduce CAR T cell production costs, shorten the vein-to-vein time, and provide resources to scale-up quickly. There are two potential options to achieving decentralization - i.e., developing a network of manufacturing plants (e.g., academic hospitals) or CAR T at the bedside by utilizing the GMP-in-a-box strategy. Decentralization of CAR $\mathrm{T}$ manufacturing is an investment-intensive approach for hospitals, on top of stringent regulatory requirements. It is now well established that academic medical centers are equipped to manufacture CAR T cells using GMP grade facilities or automated CAR T manufacturing products like the Miltenyi Prodigy system or Lonza Cocoon incubator.

With a more permissive regulatory landscape, Switzerland has started decentralized manufacturing and providing CAR T cell therapies at $\$ 150,000$ to $\$ 200,000$, approximately half the price of FDA-approved CAR T cell therapies in the USA [38]. According to the Public Health Service Act in the USA, human cells, tissues, and cellular/tissue-based products like CAR T cell therapies are regulated under section "351 product". Section 351 subjects institutions to the same regulatory and pre-market approval as drugs, whereas Section 361 is applied to all other cellular therapies like autologous and allogeneic stem cell transplants, not requiring stringent regulatory guidelines [39]. To achieve the commercialization of CAR T cell therapy utilizing the decentralization process, we will need significant regulatory changes and active collaboration between academia and pharmaceutical companies.

\section{Next Generation of Cell Therapy}

While autologous CAR T cell therapy has heralded the way to a new class of immunotherapies, they have multiple issues, including cost, variability in the product, long manufacturing 
times, and complex logistics. As mentioned earlier, decentralization and using "CAR T-cells in a box" is a good strategy; we need to improve gene-transfer tools to expedite and scale production. Non-viral approaches like transposon/ transposase-based systems provide the advantage of simplicity and cheaper production along with an even lower risk of insertional mutagenesis compared to viral vector-based technology [40].

While autologous CAR T cell therapy is growing globally, allogeneic CAR therapy is emerging as an intriguing option. "Off the Shelf" or allogeneic cellular therapies overcomes the challenges by reducing the time to treatment, ability to have high-quality starting material, and potential to reduce cost [41]. There are two main problems with allogeneic CAR therapy, the first being the risk of graft versus host disease. Investigators have adapted strategies like $\mathrm{T}$ cell subset selection (e.g., $\gamma \delta \mathrm{T}$ cells) or gene-editing (e.g., use of CRISPR/Cas9 or zinc finger nucleases to knock down TCR $\alpha \beta$ and/or MHC I in donor cells and produce universal CAR T cells). The second issue is immunological rejection leading to limited of in vivo expansion and persistence of allogeneic CAR cells. To overcome these issues, scientists have tried to incorporate more intense lymphodepletion chemotherapy or genetic modification to remove donor major histocompatibility complex [42].

Non-T cell-based cellular therapies like CAR-NK cells provide an avenue to overcome the challenges by its dual killingcapacity, i.e., CAR-dependent and -independent mechanisms, off the shelf approach with fewer side effects and ability to treat non-hematological malignancies. As the field of cellular therapies expands, the next generation of CAR therapy is continuing to focus on reducing toxicity, improving efficacy while expanding to solid cancer and non-oncological indications.

\section{Location of Care}

It is well established that outpatient administration of stem cell transplants can lead to cost-reduction and provide better HRQoL and PRO [43]. In the TRANSCEND NHL 001 study, liso-cel demonstrated an improvement in HRQoL post 1 year of treatment [44]. Outpatient administration of CAR T cell therapy may further lead to improved reimbursement and less strain on the inpatient bed utilization, especially during a pandemic. Lyman et al. developed a model to study CAR T cell therapy's economic evaluation based on site of care, including inpatient vs. outpatient and academic vs. nonacademic settings in patients with $\mathrm{R} / \mathrm{R}$ large cell lymphoma [45]. Administration of CAR T cell therapy in nonacademic specialty oncology networks compared to academic centers was associated with a $\$ 29,834(55.9 \%)$ decrease in hospitalization along with a $\$ 3154(20.1 \%)$ reduction in procedure cost. With a clear advantage in reducing the cost of care, we face the challenge of making these effective therapies available to patients globally.
First and foremost, the toxicity profile has to be conducive for the outpatient delivery of cellular therapy. Bachier et al. demonstrated the safety of outpatient administration of lisocel data on 44 patients with R/R Large B cell lymphoma treated in three clinical trials: TRANSCEND NHL 001 (NCT02631044), PILOT (NCT03483103), and OUTREACH (NCT03744676). Of these 44 patients, $45 \%$ of patients remained outpatient, and only $5 \%$ needed intensive care unit utilization [46]. Secondly, institutions need to have proper infrastructure and personnel to help perform outpatient therapy. Telemedicine has been transformative. Various organizations have shared their experience of implementing outpatient CAR T administration [47]. These standardized operating procedures and education material can provide critical help to establish outpatient cellular therapy.

Outpatient administration of cellular therapies has a potential to reduce hospital burden by shifting the inpatient volume in outpatient setting especially during unprecedented times like the ongoing pandemic; however, this may put patients at excess financial strain. It has been demonstrated that outpatient-based stem cell transplantation may lead to costshifting with more outpatient facility charges and increased out-of-pocket cost for patients [48]. With an exploding field of cellular therapies and more efficacious/safer therapies being actively developed, we need to focus on therapies that provide value to our patient by balancing efficacy and cost.

\section{Patient-Related Factors}

\section{Wide-Scale Applicability}

As previously discussed, a significant number of patients who are potentially eligible for CAR T cell therapy are not receiving a lifesaving option. An important modifiable factor includes the safety and utility of CAR T cell therapy in a broad age group [49-52]. Given the risk of CRS and ICANS, CAR $T$ cell therapy has generally been restricted to relatively younger and fit patients. Real-world outcomes of CAR T cell therapy have been crucial in understanding outcomes in patients $>$ 65 years, showing safety and similar efficacy in patients with $\mathrm{R} / \mathrm{R}$ large cell lymphoma. With the anticipated approval of CAR T cells in multiple myeloma, a disease with an average age of diagnosis at 70 , developing CAR T products that are safe and effective is essential [53].

\section{Financial and Social Support}

Patients and caregivers undergoing CAR T cell therapy have unique challenges, including logistics of care, transportation issues, and the social and emotional burden of treatment on top of the physical effects of the treatment itself. Centers have implemented a multidisciplinary evaluation from geriatricians, physical and occupational therapy, social workers, and 
dieticians before stem cell transplants to not only select appropriate patients but act on modifiable risk factors [54]. Other centers have implemented remote monitoring, utilizing the digital platform for patient education to identify barriers to access and study patient-reported outcomes [55]. For improved access, it is critical for a holistic approach for patients undergoing CAR T cell therapy.

\section{Patient-Reported Outcomes (PRO)}

Balancing disease control with quality of life is a cornerstone in improving access to CAR T cell therapy [32]. In patients with relapsed large cell lymphoma, both Axi-cel and Liso-cel have shown improvement in health-related quality of life (HRQoL). JULIET study reported a meaningful improvement in QOL at 12- and 18-months using Functional Assessment of Cancer Therapy-Lymphoma (FACT-Lym) and Short Form36 (SF-36) health survey [56]. Similarly, patients treated with liso-cel have improved HRQoL at 6 and 12 months using the European Organization for Research and Treatment of Cancer quality of life questionnaire C30 questionnaire (EORTC QLQ-C30) [44]. Recently, HRQoL in patients treated with idecabtagene vicleucel (ide-cel) was reported at the American Society of Hematology. From the registration Karmma trial, EORTC QLQ-C30 and EuroQol 5 dimensions 5 level instruments demonstrated clinically meaningful improvement from baseline at months 2 through 15 [57, 58••]. Core domains of PRO like physical functioning, diseaserelated symptoms, and adverse events are critical elements to effective therapy. Future development and regulatory approval of CAR T cell development should focus on treatments that continue to improve HRQoL on top of clinical outcomes.

\section{Cost-Effectiveness and Affordability of CAR T Cell Therapy}

Cost of care and reimbursement challenges has been a persistent issue since the first FDA approval of CAR T cell products in 2017. Since then, we have seen a steady improvement in reimbursement strategies by the CMS. There are two primary payment components - i.e., Medicare Severity-Diagnosis Related Group (MS-DRG) and New Technology Add on Payment (NTAP). The following are the changes since 2018:

1) CAR T cell-specific MS-DRG. Initially, in October 2017, a non-specific MS-DRG was introduced with payment between $\$ 6000$ and $\$ 16,000$. Between October 1, 2018, and September 30, 2020, MS-DRG 016 was utilized to pay $\$ 43,094$. Most recently, on October 1, 2020, MS-DRG 018 was introduced with an unadjusted base payment rate of $\$ 239,929$.

2) NTAP. This option provides an additional payment to hospitals above the standard MS-DRG. Between
October 1, 2018, and September 30, 2019, NTAP was approved at a $50 \%$ cap of $\$ 186,000$, which was increased to $\$ 242,500$ in 2019-2020. In the most recent updates on October 1, 2020, CMS has discontinued NTAP status on all approved CAR T cell products (Kymriah, Yescarta, Tecartus, and Beryanzi) [59].

As noted by the American Society of Clinical Oncology, the average cost of a CAR-T cell product at $\$ 373,000$, with the estimated cost of CAR-T cell therapy and related services at $\$ 419,238$; these new reimbursement programs still fall short of the total cost of care [60].

With the high cost of therapy and related costs, it is imperative to understand CAR T cell therapy's cost-effectiveness. Quality-adjusted life years (QALYs) are commonly utilized to assess therapy value in the USA. Multiple studies have reported Tisa-cel and Axi-Cel's cost-effectiveness compared to the standard of care for these therapies' FDA-approved indications $[61,62]$. One consistent finding from these reported studies is that a significant improvement in overall survival over the standard of care is required for CAR T cell therapies to demonstrate an acceptable value level. As we study cost of therapy, it is imperative we study life-time cost of care especially improving patient outcomes and increasing amount of novel but expensive therapies being FDA approved.

Medicare reimbursements rates and manufacturer prices for CAR $T$ cell therapy raise significant concerns on CAR T-centers' financial viability. There is an urgent need for manufacturer price reduction and novel reimbursement strategies to offset losses [63]. Two payment mechanisms are being evaluated - milestone-based contracts $(\mathrm{MBC})$ and outcome-based agreements (OBA). After Novartis received FDA approval for Kymriah for R/R B-ALL, they introduced an optional OBA program. The provider will not be billed unless patients achieve full remission 35th day after CAR T cell infusion. European countries developed individual reimbursement schemes for CAR T cell therapy, e.g., Germany has outcome-based rebates that are linked to individual patient outcomes and Italy has outcome-based staged payment, where payments are giving in three installments linked to individual patient outcomes. Compared to Germany and Italy, the UK has coverage of CAR T cell with future price reassessment based on longer-term follow-up and post-launch data [64]. With a growing market of cellular therapies, both payers and providers will need to transition from single patient/case agreements to some form of global episode-based care.

\section{Summary}

CAR T cell therapy has been revolutionary for treating cancers, with more than 900 cellular therapy trials expected to be available between 2020 and 2025 . However, the current 
landscape of CAR T cell therapies in hematologic malignancies, though promising, lacks the ability to optimize access. The current CAR T therapies are resource intensive and prohibitively expensive. Balancing improved access while maintaining high-quality safe and good outcomes will allow this field to further meet patient demand. In this article, we have outlined the current challenges and provided solutions to bridge the gap to gap between today's landscape and the potential for the future with CAR T cell therapies.

\section{Declarations}

Conflict of Interest Praveen Ramakrishnan Geethakumari declares that he has no conflict of interest.

Dheepthi Perumal Ramasamy declares that she has no conflict of interest.

Bhagirathbhai Dholaria has received research funding from Takeda, Poseida Therapeutics, Janssen, and Angiocrine Bioscience, and has served on an advisory board for Jazz Pharmaceuticals.

Jesús Berdeja has received research funding (paid in his name to his institution) from AbbVie, Amgen, Acetylon, bluebird bio, Bristol-Myers Squibb, Celgene, Celularity, Constellation Pharmaceuticals, CRISPR Therapeutics, Curis, EMD Serono, Genentech, Glenmark Pharmaceuticals, Ichnos Sciences, Janssen, Kesios Therapeutics, Eli Lilly, Novartis, Poseida Therapeutics, Sanofi, Takeda, Teva, and Vivolux; and has served as a consultant for Amgen, Bioclinica, bluebird bio, Bristol-Myers Squibb, Celgene, CRISPR Therapeutics, Janssen, Karyopharm Therapeutics, Kite Pharma, Legend Biotech, Prothena, Secura Bio, and Laboratoires Servier, with all compensation made payable to his institution.

Ankit Kansagra has participated on advisory boards for Alnylam Pharmaceuticals, Bristol-Myers Squibb/Celgene, GlaxoSmithKline, Karyopharm Therapeutics, Oncopeptides, Pharmacyclics, Pfizer, Takeda, and Sanofi.

Human and Animal Rights and Informed Consent This article does not contain any studies with human or animal subjects performed by any of the authors.

\section{References}

Papers of particular interest, published recently, have been highlighted as:

- Of importance

-• Of major importance

1. Waldman AD, Fritz JM, Leonardo MJ. A guide to cancer immunotherapy: from $\mathrm{T}$ cell basic science to clinical practice. Nat Rev Immunol. 2020;20:651-68.

2. June $\mathrm{CH}$, Sadelain M. Chimeric antigen receptor therapy. N Engl J Med. 2018;379(1):64-73. https://doi.org/10.1056/ NEJMra1706169.

3.• Maude SL, Laetsch TW, Buechner J, et al. Tisagenlecleucel in children and young adults with B-cell lymphoblastic leukemia. N Engl J Med. 2018;378(5):439-48. https://doi.org/10.1056/ NEJMoa1709866 Pivotal study of CAR T-cell therapy in pediatric acute lymphoblastic leukemia.
4. Neelapu SS, Locke FL, Bartlett NL, et al. Axicabtagene ciloleucel CAR T-cell therapy in refractory large B-cell lymphoma. N Engl J Med. 2017;377(26):2531-44. https://doi.org/10.1056/ NEJMoa1707447. Pivotal study of CAR T-cell therapy in adults with relapsed/refractory Large Cell Lymphoma.

5. Schuster SJ, Bishop MR, Tam CS, Waller EK, Borchmann P, McGuirk JP, et al. Tisagenlecleucel in adult relapsed or refractory diffuse large B-cell lymphoma. N Engl J Med. 2019;380(1):45-56. https://doi.org/10.1056/NEJMoa1804980.

6. Abramson JS, Palomba ML, Gordon LI, Lunning MA, Wang M, Arnason $\mathrm{J}$, et al. Lisocabtagene maraleucel for patients with relapsed or refractory large B-cell lymphomas (TRANSCEND NHL 001): a multicentre seamless design study. Lancet. 2020;396(10254):839-52. https://doi.org/10.1016/S01406736(20)31366-0.

7. Wang M, Munoz J, Goy A, Locke FL, Jacobson CA, Hill BT, et al. KTE-X19 CAR T-cell therapy in relapsed or refractory mantle-cell lymphoma. N Engl J Med. 2020;382(14):1331-42. https://doi.org/ 10.1056/NEJMoa1914347.

8. Wierda WG, Dorritie KA, Munoz J, et al. Transcend CLL 004: phase 1 cohort of lisocabtagene maraleucel (liso-cel) in combination with ibrutinib for patients with relapsed/refractory (R/R) chronic lymphocytic leukemia/small lymphocytic lymphoma (CLL/ SLL). Blood. 2020;136(Supplement 1):39-40. https://doi.org/10. 1182/blood-2020-140622.

9. Jacobson C, Chavez JC, Sehgal AR, et al. Primary analysis of Zuma-5: a phase 2 study of axicabtagene ciloleucel (Axi-Cel) in patients with relapsed/refractory (R/R) indolent non-Hodgkin lymphoma (iNHL). Blood. 2020;136(Supplement 1):40-1. https://doi. org/10.1182/blood-2020-136834.

10. Brudno JN, Maric I, Hartman SD, et al. T cells genetically modified to express an anti-B-cell maturation antigen chimeric antigen receptor cause remissions of poor-prognosis relapsed multiple myeloma. J Clin Oncol. 2018;36(22):2267-80.

11. Xu J, Chen LJ, Yang SS, Sun Y, Wu W, Liu YF, et al. Exploratory trial of a biepitopic CAR T-targeting B cell maturation antigen in relapsed/refractory multiple myeloma. Proc Natl Acad Sci U S A. 2019;116(19):9543-51. https://doi.org/10.1073/pnas.1819745116.

12. Gottlieb S, Marks P.Statement from FDA commissioner Scott Gottlieb, M.D. and Peter Marks, M.D., Ph.D., director of the Center for Biologics Evaluation and Research on new policies to advance development of safe and effective cell and gene therapies. http:/www.fda.gov/news-events/press-announcements/statementfda-commissioner-scott-gottlieb-md-and-peter-marks-md-phddirector-center-biologics. Accessed 15 Apr 2021.

13. Jaglowski S, Hu Z-H, Zhang Y, et al. Tisagenlecleucel chimeric antigen receptor (CAR) T-cell therapy for adults with diffuse large B-cell lymphoma (DLBCL): real world experience from the Center for International Blood \& Marrow Transplant Research (CIBMTR) Cellular Therapy (CT.) Registry. Blood. 2019;134(Supplement_1): 766. https://doi.org/10.1182/blood-2019-130983.

14. Locke FL, Ghobadi A, Jacobson CA, Miklos DB, Lekakis LJ, Oluwole OO, et al. Long-term safety and activity of axicabtagene ciloleucel in refractory large B-cell lymphoma (ZUMA-1): a singlearm, multicentre, phase 1-2 trial. Lancet Oncol. 2019;20(1):31-42. https://doi.org/10.1016/S1470-2045(18)30864-7.

15. Pasquini MC, Locke FL, Herrera AF, Siddiqi T, et al. Postmarketing use outcomes of an anti-CD19 chimeric antigen receptor (CAR) T cell therapy, axicabtagene ciloleucel (Axi-Cel), for the treatment of large B cell lymphoma (LBCL) in the United States (US). Blood. 2019;134(Supplement 1):764. https://doi.org/10. 1182/blood-2019-124750.

16. Nastoupil LJ, Jain MD, Feng L, Spiegel JY, Ghobadi A, Lin Y, et al. Standard-of-care axicabtagene ciloleucel for relapsed or refractory large B-cell lymphoma: results from the US lymphoma 
CAR T consortium. J Clin Oncol. 2020;38(27):3119-28. https:// doi.org/10.1200/JCO.19.02104.

17. Jacobson CA, Hunter BD, Redd R, Rodig SJ, Chen PH, Wright K, et al. Axicabtagene ciloleucel in the non-trial setting: outcomes and correlates of response, resistance, and toxicity. J Clin Oncol. 2020;38(27):3095-106. https://doi.org/10.1200/JCO.19.02103.

18. Fiorenza S, Ritchie DS, Ramsey SD, Turtle CJ, Roth JA. Value and affordability of CAR T-cell therapy in the United States. Bone Marrow Transplant. 2020;55:1706-15. https://doi.org/10.1038/ s41409-020-0956-8.

19. Institute for Clinical and Economic Review: Chimeric antigen receptor T-cell therapy for B-cell cancers: effectiveness and valuefinal evidence report. https://icer-review.org/wpcontent/uploads/ 2017/07/ICER_CAR_T_Final_Evidence_Report_032318.pdf. Accessed 15 Apr 2021.

20. Lin JK, Lerman BJ, Barnes JI, Boursiquot BC, Tan YJ, Robinson $\mathrm{AQL}$, et al. Cost effectiveness of chimeric antigen receptor T-cell therapy in relapsed or refractory pediatric B-cell acute lymphoblastic leukemia. J Clin Oncol. 2018;36(32):3192-202. https://doi.org/ 10.1200/JCO.2018.79.0642.

21.• Lin JK, Muffly LS, Spinner MA, Barnes JI, Owens DK, GoldhaberFiebert JD. Cost effectiveness of chimeric antigen receptor T-cell therapy in multiply relapsed or refractory adult large B-cell lymphoma. J Clin Oncol. 2019;37(24):2105-19. https://doi.org/10. $1200 / \mathrm{JCO} .18 .02079$ Key article describing the cost effectiveness of CAR T-cell therapy in lymphoma.

22. Kilgore KM, Mohammadi I, Schroeder A, et al. Medicare patients receiving chimeric antigen receptor T-cell therapy for non-Hodgkin lymphoma: a first real-world look at patient characteristics, healthcare utilization and costs. Blood. 2019;134(Supplement_1): 793. https://doi.org/10.1182/blood-2019-124364.

23. Silbert S, Yanik GA, Shuman AG. How should we determine the value of CAR T-cell therapy? AMA J Ethics. 2019;21(10):E844 51. Published 2019 October 1. https://doi.org/10.1001/amajethics. 2019.844.

24.• Komanduri KV. Chimeric antigen receptor T-cell therapy in the management of relapsed non-Hodgkin lymphoma. J Clin Oncol. 2021;39(5):476-86. https://doi.org/10.1200/JCO.20.01749 Clinically focused article on utilization of FDA approved CAR T cell therapy in large cell lymphoma.

25. Reagan PM, Neelapu SS. How I manage: pathophysiology and management of toxicity of chimeric antigen receptor T-cell therapies. J Clin Oncol. 2021;39(5):456-66.

26. Berdeja JG. Practical aspects of building a new immunotherapy program: the future of cell therapy. Hematology Am Soc Hematol Educ Program. 2020;1:579-84.

27. Majhail NS. Optimizing quality and efficiency of healthcare delivery in hemeatopoietic cell transplantation. Curr Hematol Malig Rep. 2015;10:199-204.

28. Maus MV, Nikiforow S. The why, what and how of the new FACT standards for immune effector cells. J Immunother Cancer. 2017;5: 36.

29. Porter ME. What is value in health care? N Engl J Med. 2010;363(26):2477-81.

30. Cornetta K, Komanduri KV. Regulatory issues in gene-modified immune effector cell therapy. In: Lee DW, Shah NN, editors. Chimeric antigen receptor T-cell therapies for cancer E-book: a practical guide. Cambridge: Elsevier health Sciences; 2019. p. 209-22.

31. Lymphoma based on insurance status (public versus private) and distance traveled to treatment center. tct.confex.com. Published Februay 9, 2021. https://tct.confex.com/tct/2021/meetingapp.cgi/ Paper/16691. Accessed February 22, 2021.

32. Chakraborty R, Sidana S, Shah GL, Scordo M, Hamilton BK, Majhail NS. Patient-reported outcomes with chimeric antigen receptor T cell therapy: challenges and opportunities. Biol Blood
Marrow Transplant. 2019;25(5):e155-62. https://doi.org/10.1016/ j.bbmt.2018.11.025.

33. Elnair R, Schmit-Pokorny K, Jourdan D, et al. Assesment of time to CAR-T cell therapy and patients' outcomes in relapsed or refractory diffuse large B-cell lymphoma based on insurance status (public versus private) and distance traveled to treatment center. tct. confex.com. Published Februay 9, 2021. https://tct.confex.com/ tct/2021/meetingapp.cgi/Paper/16691. Accessed February 22, 2021.

34. Ruark J, Mullane E, Cleary N, Cordeiro A, Bezerra ED, Wu V, et al. Patient-reported neuropsychiatric outcomes of long-term survivors after chimeric antigen receptor T cell therapy. Biol Blood Marrow Transplant. 2020;26(1):34-43. https://doi.org/10.1016/j. bbmt.2019.09.037.

35. Kansagra A, Farnia S, Majhail N. Expanding access to chimeric antigen receptor T-cell therapies: challenges and opportunities. Am Soc Clin Oncol Educ Book. 2020;40:1-8. https://doi.org/10. 1200/EDBK 279151.

36. Lutfi F, Moaath Mustafa A, Siglin J, et al. The impact of bridging therapy prior to CAR-T cell therapies clincial outcomes of patients with relapsed refractory large B-cell lymphoma. Tct.fonfex.com. Published February 8, 2021. https://tct.confex.com/tct/2021/ meetingapp.cgi/Paper/16887. Accessed February 22, 2021.

37. Hua J, Baoxia D, Gao L, et al. Clinical results of multicenter study of the first-in-human dual BCMA and CD19 targeted novel platform fast CAR-T cell therapy for patients with relapsed/refractory multiple myeloma. ash.confex.com. Published December 5, 2020. https://ash.confex.com/ash/2020/webprogram/Paper138614.html. Accessed February 22, 2021.

38. Hoffman A. Swiss to take on big pharma with cheaper cancer treatment. Bloomberg.com. https://www.bloomberg.com/news/articles/ 2019-07-28/swiss-to-take-on-big-pharma-with-cheaper-cancertreatment-nzz. Published July 28, 2019. Accessed December 13, 2020.

39. Rej R. US food and drug administration, center for devices and radiological health. Clin Chem. 2003;49(5):840-1. http://www. fda.gov/cdrh. Accessed 15 Apr 2021.

40. Caldwell KJ, Gottschalk S, Talleur AC. Allogeneic CAR cell therapy-more than a pipe dream. Front Immunol. 2020;11: 618427. https://doi.org/10.3389/fimmu.2020.618427.

41. Holbro A, Ahmad I, Cohen S, Roy J, Lachance S, Chagnon M, et al. Safety and cost-effectiveness of outpatient autologous stem cell transplantation in patients with multiple myeloma. Biol Blood Marrow Transplant. 2013;19(4):547-51. https://doi.org/10.1016/j. bbmt.2012.12.006.

42. Townsend MH, Bennion K, Robison RA, O'Neill KL. Paving the way towards universal treatment with allogenic T cells. Immunol Res. 2020;68(1):63-70. https://doi.org/10.1007/s12026-02009119-7.

43. Magnani CF, Tettamanti S, Alberti G, et al. Transposon-based CAR T cells in acute leukemias: where are we going? Cells. 2020;9(6): 1337. Published 2020 May 27. https://doi.org/10.3390/ cells9061337.

44. Patrick DL, Powers A, Parisi M, Kim Y, Garcia J, Dehner C, et al. Impact of lisocabtagene maraleucel (liso-cel) treatment on healthrelated quality of life and health utility in patients (pts) with relapsed/refractory (R/R) aggressive $\mathrm{B}$ cell non-Hodgkin lymphoma (NHL): Transcend NHL 001. Blood. 2019;134(Supplement 1): 66. https://doi.org/10.1182/blood-2019-127145.

45. Lyman GH, Nguyen A, Snyder S, Gitlin M, Chung KC. Economic evaluation of chimeric antigen receptor T-cell therapy by site of care among patients with relapsed or refractory large B-cell lymphoma [published correction appears in JAMA Netw Open. 2020;3(4):e208117]. JAMA Netw Open 2020;3(4):e202072 Published 2020 Apr 1. https://doi.org/10.1001/jamanetworkopen. 2020.2072 . 
46. Bachier CR, Godwin JE, Andreadis C, et al. Outpatient treatment with lisocabtagene maraleucel (liso-cel) across a variety of clinical sites from three ongoing clinical studies in relapsed/refractory $(\mathrm{R} / \mathrm{R})$ large B-cell lymphoma (LBCL). J Clin Oncol. 2020;38(suppl 15): 8037. https://doi.org/10.1200/JCO.2020.38.15 suppl.8037.

47. Vaidya R, Seegars MB, Rodriguez C, et al. Development and implementation of outpatient CAR-T program at the Wake Forest Baptist Comprehensive Cancer Center. tct.confex.com. Publishsed February 8, 2021. https://tct.confex.com/tct/2021/meetingapp.cgi/ Paper/17660. Accessed February 11, 2021.

48. Rizzo JD, Vogelsang GB, Krumm S, Frink B, Mock V, Bass EB. Outpatient-based bone marrow transplantation for hematologic malignancies: cost saving or cost shifting? J Clin Oncol. 1999;17(9): 2811-8. https://doi.org/10.1200/JCO.1999.17.9.2811.

49. Bishop MR. The benefit of CAR T cells in older patients. Blood. 2020;135(23):2020-1. https://doi.org/10.1182/blood.2020005592.

50. Zettler ME, Feinberg BA, Phillips EG Jr, Klink AJ, Mehta S, Gajra A. Real-world adverse events associated with CAR T-cell therapy among adults age $\geq 65$ years [published online ahead of print, 2020 August 11]. J Geriatr Oncol. 2020;S1879-4068(20):30145-4. https://doi.org/10.1016/j.jgo.2020.07.006.

51. Ram R, Shargian L, Bar-On Y, et al. Toxicity and efficacy of CAR$\mathrm{T}$ in patients with DLBCL above the age of 70 years compare to younger patients - a matched control multi-center cohort study. tct. confex.com. Published Februay 10, 2021, 2021.https://tct.confex. com/tct/2021/meetingapp.cgi/Paper/16814. Accessed February 22.

52. Pasquini M, Locke F, Al H, et al. Post-marketing use outcomes of an anti-CD19 chimeric antigen receptor (CAR) T cell therapy, axicabtagene ciloleucel (Axi-Cel), for the treatment of large B cell lymphoma (LBCL) in the United States. Blood. 2019;134(Supplement_1):764. https://doi.org/10.1182/blood2019-124750.

53. Berdeja J, Noopur R, Siegel S, et al. Efficacy and safety of idecabtagene icleucel (ide-cel, bb2121) in elderly patients with relapsed and refractory multiple myeloma: KarMMa subgroup analysis. ash.confex.com. Published December 5, 2020. https://ash. confex.com/ash/2020/webprogram/Paper134322.html. Accessed February 11, 2021.

54. BA KK, Molloy E, et al. Recommendations and outcomes from a geriatric assessment guided multidisciplinary clinic prior to autologous stem cell transplant in older patients [published online ahead of print, 2020 November 5]. J Geriatr Oncol. 2020;S18794068(20):30491-4. https://doi.org/10.1016/j.jgo.2020.10.019.

55. Matthews A, Sidana S, Seymor L, et al. QIM19-136: Developing an ideal CAR-T cell therapy patient experience through humancentered design and innovation. J Natl Compr Canc Netw. 17(3.5):QIM19-136. https://jnccn.org/view/journals/jnccn/17/3.5/ article-pQIM19-136.xml. Accessed 28 Feb 2021.
56. Maziarz RT, Waller EK, Jaeger U, Fleury I, McGuirk J, Holte H, et al. Patient-reported long-term quality of life after tisagenlecleucel in relapsed/refractory diffuse large B-cell lymphoma. Blood Adv. 2020;4(4):629-37. https://doi.org/10.1182/bloodadvances. 2019001026.

57. Shah N, Delforge M, San-Miguel J, et al. Secondary quality-of-life domains in patients with relapse and refractory multiple myeloma treated with the Bcma-directed CAR T cell therapy idecabtagene vicleucel (ide-cel; bb2121): results from the Karmma Clinical Trial. ash.confex.com. Published December 6, 2020: https://ash.confex. com/ash/2020/webprogram/Paper136665.html. Accessed February 11, 2021.

58.• Munshi NC, Anderson LD Jr, Shah N, et al. Idecabtagene vicleucel in relapsed and refractory multiple myeloma. N Engl J Med. 2021;384(8):705-16. https://doi.org/10.1056/NEJMoa2024850 Pivotal study of CAR T-cell therapy in multiple myeloma.

59. CAR T-cell therapy: an update on coverage and reimbursement. CAR T-cell therapy: an update on coverage and reimbursement Hematology.org. https://www.hematology.org/advocacy/policynews/2019/car-t-cell-therapy-an-update-on-coverage-andreimbursement. Published August 14, 2019. Accessed December 13,2020 .

60. https://www.asco.org/sites/new-www.asco.org/files/content-files/ advocacy-and-policy/documents/2020-CARTPolicyBrief-Update. pdf. Accessed March 14, 2021.

61. Whittington MD, McQueen RB, Ollendorf DA, et al. Long-term survival and cost-effectiveness associated with axicabtegene ciloleuccel vs. chemotherapy for treatment of B-cell lymphoma. JAMA Netw Open. 2019;2(2):e190035. Published 2019 Feb 1. https://doi.org/10.1001/jamanetworkopen.2019.0035.

62. Sarkar RR, Gloude NJ, Schiff D, Murphy JD. Cost-effectiveness of chimeric antigen receptor T-Cell therapy in pediatric relapsed/ refractory B-cell acute lymphoblastic leukemia. J Natl Cancer Inst. 2019;111(7):719-26. https://doi.org/10.1093/jnci/djy193.

63. Leech AA, Neumann PJ, Cohen JT, Jagasia M, Dusetzina SB. Balancing value with affordability: cell immunotherapy for cancer treatment in the US. Oncologist. 2020;25(7):e1117-9. https://doi. org/10.1634/theoncologist.2020-0025.

64. Jørgensen J, Hanna E, Kefalas P. Outcomes-based reimbursement for gene therapies in practice: the experience of recently launched CAR-T cell therapies in major European countries. J Mark Access Health Policy. 2020;8(1):1715536. Published 2020 January 15. https://doi.org/10.1080/20016689.2020.1715536.

Publisher's Note Springer Nature remains neutral with regard to jurisdictional claims in published maps and institutional affiliations. 